\title{
Some classes of sequence spaces defined by a Musielak-Orlicz function
}

\author{
Sunil K. Sharma \\ Department of Mathematics, \\ Cluster University of Jammu, \\ Jammu, J\&K 180001, India \\ email: sunilksharma42@gmail.com
}

\begin{abstract}
In the present paper we introduce the sequence spaces $c_{0}\{\mathcal{M}, \Lambda, p, q\}$, $c\{\mathcal{M}, \Lambda, p, q\}$ and $l_{\infty}\{\mathcal{M}, \Lambda, p, q\}$ defined by a Musielak-Orlicz function $\mathcal{M}=\left(M_{\mathrm{k}}\right)$. We study some topological properties and prove some inclusion relations between these spaces.
\end{abstract}

\section{Introduction and preliminaries}

An Orlicz function $M:[0, \infty) \rightarrow[0, \infty)$ is a continuous, non-decreasing and convex function such that $M(0)=0, M(x)>0$ for $x>0$ and $M(x) \longrightarrow \infty$ as $x \longrightarrow \infty$.

Lindenstrauss and Tzafriri [3] used the idea of Orlicz function to define the following sequence space,

$$
\ell_{M}=\left\{x \in w: \sum_{k=1}^{\infty} M\left(\frac{\left|x_{k}\right|}{\rho}\right)<\infty\right\}
$$

which is called as an Orlicz sequence space. Also $\ell_{M}$ is a Banach space with the norm

$$
\|x\|=\inf \left\{\rho>0: \sum_{k=1}^{\infty} M\left(\frac{\left|x_{k}\right|}{\rho}\right) \leq 1\right\} .
$$

2010 Mathematics Subject Classification: 40A05, 46A45, 46E30

Key words and phrases: Orlicz function, Musielak-Orlicz function, paranorm space, sequence space 
Also, it was shown in [3] that every Orlicz sequence space $\ell_{M}$ contains a subspace isomorphic to $\ell_{p}(p \geq 1)$. The $\Delta_{2}$ - condition is equivalent to $M(L x) \leq$ $\mathrm{LM}(\mathrm{x})$, for all $\mathrm{L}$ with $0<\mathrm{L}<1$. An Orlicz function $M$ can always be represented in the following integral form

$$
M(x)=\int_{0}^{x} \eta(t) d t
$$

where $\eta$ is known as the kernel of $M$, is right differentiable for $t \geq 0, \eta(0)=$ $0, \eta(t)>0, \eta$ is non-decreasing and $\eta(t) \rightarrow \infty$ as $t \rightarrow \infty$.

A sequence $\mathcal{M}=\left(M_{k}\right)$ of Orlicz function is called a Musielak-Orlicz function (see [4], [8]). A sequence $\mathcal{N}=\left(\mathrm{N}_{k}\right)$ defined by

$$
\mathrm{N}_{\mathrm{k}}(v)=\sup \left\{|v| u-M_{k}(u): u \geq 0\right\}, k=1,2, \cdots
$$

is called the complementary function of a Musielak-Orlicz function $\mathcal{M}$. For a given Musielak-Orlicz function $\mathcal{M}$, the Musielak-Orlicz sequence space $t_{\mathcal{M}}$ and its subspace $h_{\mathcal{M}}$ are defined as follows

$$
\begin{gathered}
\mathrm{t}_{\mathcal{M}}=\left\{x \in w: I_{\mathcal{M}}(c x)<\infty \text { for some } c>0\right\}, \\
h_{\mathcal{M}}=\left\{x \in w: I_{\mathcal{M}}(c x)<\infty \text { for all } c>0\right\},
\end{gathered}
$$

where $\mathrm{I}_{\mathcal{M}}$ is a convex modular defined by

$$
\mathrm{I}_{\mathcal{M}}(\mathrm{x})=\sum_{\mathrm{k}=1}^{\infty} \mathrm{M}_{\mathrm{k}}\left(\mathrm{x}_{\mathrm{k}}\right), \mathrm{x}=\left(\mathrm{x}_{\mathrm{k}}\right) \in \mathrm{t}_{\mathcal{M}} .
$$

We consider $t_{\mathcal{M}}$ equipped with the Luxemburg norm

$$
\|x\|=\inf \left\{k>0: I_{\mathcal{M}}\left(\frac{x}{k}\right) \leq 1\right\}
$$

or equipped with the Orlicz norm

$$
\|x\|^{0}=\inf \left\{\frac{1}{k}\left(1+I_{\mathcal{M}}(k x)\right): k>0\right\} .
$$

Let $w, l_{\infty}, c$ and $c_{0}$ denote the spaces of all, bounded, convergent and null sequences $x=\left(x_{k}\right)$ with complex terms respectively. The zero sequence $(0,0, \ldots)$ is denoted by $\theta$ and $p=\left(p_{k}\right)$ is a sequence of strictly positive real numbers. Further the sequence $\left(p_{k}^{-1}\right)$ will be represented by $\left(t_{k}\right)$. 
Mursaleen and Noman [6] introduced the notion of $\lambda$-convergent and $\lambda$ bounded sequences as follows :

Let $\lambda=\left(\lambda_{k}\right)_{k=1}^{\infty}$ be a strictly increasing sequence of positive real numbers tending to infinity i.e.

$$
0<\lambda_{0}<\lambda_{1}<\cdots \text { and } \lambda_{k} \rightarrow \infty \text { as } k \rightarrow \infty
$$

and said that a sequence $x=\left(x_{k}\right) \in w$ is $\lambda$-convergent to the number L, called the $\lambda$-limit of $x$ if $\Lambda_{m}(x) \longrightarrow L$ as $m \rightarrow \infty$, where

$$
\lambda_{m}(x)=\frac{1}{\lambda_{m}} \sum_{k=1}^{m}\left(\lambda_{k}-\lambda_{k-1}\right) x_{k} .
$$

The sequence $x=\left(x_{k}\right) \in w$ is $\lambda$-bounded if $\sup _{m}\left|\Lambda_{m}(x)\right|<\infty$. It is well known [6] that if $\lim _{m} x_{m}=a$ in the ordinary sense of convergence, then

$$
\lim _{m}\left(\frac{1}{\lambda_{m}}\left(\sum_{k=1}^{m}\left(\lambda_{k}-\lambda_{k-1}\right)\left|x_{k}-a\right|\right)=0 .\right.
$$

This implies that

$$
\lim _{m}\left|\Lambda_{m}(x)-a\right|=\lim _{m}\left|\frac{1}{\lambda_{m}} \sum_{k=1}^{m}\left(\lambda_{k}-\lambda_{k-1}\right)\left(x_{k}-a\right)\right|=0
$$

which yields that $\lim _{m} \Lambda_{m}(x)=a$ and hence $x=\left(x_{k}\right) \in w$ is $\lambda$-convergent to a.

Let $X$ be a linear metric space. A function $p: X \rightarrow \mathbb{R}$ is called paranorm, if

1. $p(x) \geq 0$ for all $x \in X$,

2. $p(-x)=p(x)$ for all $x \in X$,

3. $p(x+y) \leq p(x)+p(y)$ for all $x, y \in X$,

4. if $\left(\lambda_{n}\right)$ is a sequence of scalars with $\lambda_{n} \rightarrow \lambda$ as $n \rightarrow \infty$ and $\left(x_{n}\right)$ is a sequence of vectors with $p\left(x_{n}-x\right) \rightarrow 0$ as $n \rightarrow \infty$, then $p\left(\lambda_{n} x_{n}-\lambda x\right) \rightarrow$ 0 as $n \rightarrow \infty$.

A paranorm $p$ for which $p(x)=0$ implies $x=0$ is called total paranorm and the pair $(X, p)$ is called a total paranormed space. It is well known that the metric of any linear metric space is given by some total paranorm (see [14], 
Theorem 10.4.2, pp. 183). For more details about sequence spaces (see [1], [2], [5], [7], [9], [10], [11], [12], [13]) and references therein.

Let $\mathcal{M}=\left(M_{k}\right)$ be a Musielak-Orlicz function and $p=\left(p_{k}\right)$ be a bounded sequence of positive real numbers and let $(X, q)$ be a seminormed space seminormed by $\mathrm{q}$. In the present paper, we define the following sequence spaces:

$$
\begin{aligned}
c_{0}\{\mathcal{M}, \Lambda, p, q\}= & \left\{x=\left(x_{k}\right) \in w:\left[M_{k}\left(\frac{q\left(\Lambda_{k}(x)\right)}{\rho}\right)\right]^{p_{k}} t_{k} \rightarrow 0, \text { as } k \rightarrow \infty,\right. \\
& \text { for some } \rho>0\}, \\
c\{\mathcal{M}, \Lambda, p, q\}=\left\{x=\left(x_{k}\right) \in w:\left[M_{k}\left(\frac{q\left(\Lambda_{k}(x)\right)}{\rho}\right)\right]^{p_{k}} t_{k} \rightarrow 0, \text { as } k \rightarrow \infty,\right. & \text { for some } L \in X \text { and for some } \rho>0\}
\end{aligned}
$$

and

$$
\begin{aligned}
l_{\infty}\{\mathcal{M}, \Lambda, p, q\}= & \left\{x=\left(x_{k}\right) \in w: \sup _{k}\left[M_{k}\left(\frac{q\left(\Lambda_{k}(x)\right)}{\rho}\right)\right]^{p_{k}} t_{k}<\infty,\right. \\
& \text { for some } \rho>0\} .
\end{aligned}
$$

If we take $p=\left(p_{k}\right)=1$, we have

$$
\begin{aligned}
c_{0}\{\mathcal{M}, \Lambda, q\}= & \left\{x=\left(x_{k}\right) \in w:\left[M_{k}\left(\frac{\mathrm{q}\left(\Lambda_{k}(x)\right)}{\rho}\right)\right] \rightarrow 0, \text { as } k \rightarrow \infty,\right. \\
& \text { for some } \rho>0\}, \\
c\{\mathcal{M}, \Lambda, q\}=\left\{x=\left(x_{k}\right) \in \mathcal{w}:\left[M_{k}\left(\frac{\mathrm{q}\left(\Lambda_{k}(x)-\mathrm{L}\right)}{\rho}\right)\right] \rightarrow 0, \text { as } k \rightarrow \infty,\right. & \text { for some } L \in X \text { and for some } \rho>0\}
\end{aligned}
$$

and

$l_{\infty}\{\mathcal{M}, \Lambda, q\}=\left\{x=\left(x_{k}\right) \in w: \sup _{k}\left[M_{k}\left(\frac{q\left(\Lambda_{k}(x)\right)}{\rho}\right)\right]<\infty\right.$, for some $\left.\rho>0\right\}$.

The following inequality will be used throughout the paper. If $0 \leq p_{k} \leq$ $\sup p_{k}=K, D=\max \left(1,2^{\mathrm{K}-1}\right)$ then

$$
\left|a_{k}+b_{k}\right|^{p_{k}} \leq D\left\{\left|a_{k}\right|^{p_{k}}+\left|b_{k}\right|^{p_{k}}\right\}
$$


for all $k$ and $a_{k}, b_{k} \in \mathbb{C}$. Also $|a|^{p_{k}} \leq \max \left(1,|a|^{k}\right)$ for all $a \in \mathbb{C}$.

The main aim of this paper is to study some toplogical properties and prove some inclusion relation between these spaces.

\section{Main results}

Theorem 1 If $\mathcal{M}=\left(M_{\mathrm{k}}\right)$ be a Musielak-Orlicz function and $\mathrm{p}=\left(\mathrm{p}_{\mathrm{k}}\right)$ be a bounded sequence of positive real numbers, then the spaces $c_{0}\{\mathcal{M}, \Lambda, p, q\}$, $\mathrm{c}\{\mathcal{M}, \Lambda, p, q\}$ and $l_{\infty}\{\mathcal{M}, \Lambda, p, q\}$ are linear spaces over the field of complex numbers $\mathbb{C}$.

Proof. Let $x=\left(x_{k}\right), y=\left(y_{k}\right) \in c\{\mathcal{M}, \Lambda, p, q\}$ and $\alpha, \beta \in \mathbb{C}$. Then there exist positive real numbers $\rho_{1}$ and $\rho_{2}$ such that

$$
\left[M_{k}\left(\frac{q\left(\Lambda_{k}(x)-L\right)}{\rho_{1}}\right)\right]^{p_{k}} t_{k} \rightarrow 0, \text { as } k \rightarrow \infty
$$

and

$$
\left[M_{k}\left(\frac{q\left(\Lambda_{k}(y)-L\right)}{\rho_{2}}\right)\right]^{p_{k}} t_{k} \rightarrow 0, \text { as } k \rightarrow \infty .
$$

Let $\rho_{3}=\max \left(2|\alpha| \rho_{1}, 2|\beta| \rho_{2}\right)$. Since $\left(M_{k}\right)$ is non-decreasing and convex by using inequality (1.1), we have

$$
\begin{aligned}
& {\left[M_{k}\left(\frac{q\left(\left(\alpha \Lambda_{k}(x)+\beta \Lambda_{k}(y)\right)-2 L\right)}{\rho_{3}}\right)\right]^{p_{k}} t_{k} } \\
& \leq {\left[M_{k}\left(\frac{q\left(\alpha \Lambda_{k}(x)-L\right)}{\rho_{3}}+\frac{q\left(\beta \Lambda_{k}(y)-L\right)}{\rho_{3}}\right)\right]^{p_{k}} t_{k} } \\
& \leq D \frac{1}{2^{p_{k}}}\left[M_{k}\left(\frac{q\left(\Lambda_{k}(x)-L\right)}{\rho_{1}}\right)\right]^{p_{k}} t_{k}+D \frac{1}{2^{p_{k}}}\left[M_{k}\left(\frac{q\left(\Lambda_{k}(y)-L\right)}{\rho_{2}}\right)\right]^{p_{k}} t_{k} \\
& \leq D\left[M_{k}\left(\frac{q\left(\Lambda_{k}(x)-L\right)}{\rho_{1}}\right)\right]^{p_{k}} t_{k}+D\left[M_{k}\left(\frac{q\left(\Lambda_{k}(y)-L\right)}{\rho_{2}}\right)\right]^{p_{k}} t_{k} \\
& \rightarrow 0 \text { as } k \rightarrow \infty .
\end{aligned}
$$

Thus, $\alpha x+\beta y \in c\{\mathcal{M}, \Lambda, p, q\}$. Hence $c\{\mathcal{M}, \Lambda, p, q\}$ is a linear space. Similarly, we can prove $c_{0}\{\mathcal{M}, \Lambda, p, q\}$ and $l_{\infty}\{\mathcal{M}, \Lambda, p, q\}$ are linear spaces over the field of complex numbers $\mathbb{C}$. 
Theorem $2 \mathcal{M}=\left(M_{\mathrm{k}}\right)$ be a Musielak-Orlicz function and $\mathrm{p}=\left(\mathrm{p}_{\mathrm{k}}\right)$ be a bounded sequence of positive real numbers, then $l_{\infty}\{\mathcal{M}, \Lambda, p, q\}$ is a paranormed space with the paranorm defined by

$$
g(x)=q\left(x_{1}\right)+\inf \left\{\rho^{\frac{p_{k}}{H}}: \sup _{k \geq 1}\left\{M_{k}\left(\frac{q\left(\Lambda_{k}(x)\right)}{\rho}\right) t_{k}^{\frac{1}{p_{k}}}\right\} \leq 1, \quad \rho>0 \text { bigg }\right\},
$$

where $\mathrm{H}=\max (1, \mathrm{~K})$.

Proof. (i) Clearly, $g(x) \geq 0$ for $x=\left(x_{k}\right) \in l_{\infty}\{\mathcal{M}, \Lambda, p, q\}$. Since $M_{k}(0)=0$, we get $g(\theta)=0$.

(ii) $g(-x)=g(x)$

(iii) Let $x=\left(x_{k}\right), y=\left(y_{k}\right) \in l_{\infty}\{\mathcal{M}, \Lambda, p, q\}$, then there exist $\rho_{1}, \rho_{2}>0$ such that

$$
\sup _{k \geq 1}\left\{M_{k}\left(\frac{q\left(\Lambda_{k}(x)\right)}{\rho_{1}}\right) t_{k}^{\frac{1}{p_{k}}}\right\} \leq 1
$$

and

$$
\sup _{k \geq 1}\left\{M_{k}\left(\frac{q(\Lambda(y))}{\rho_{2}}\right) t_{k}^{\frac{1}{p_{k}}}\right\} \leq 1
$$

Let $\rho=\rho_{1}+\rho_{2}$, then by Minkowski's inequality, we have

$$
\begin{aligned}
\sup _{k \geq 1}\left\{M_{k}\left(\frac{q\left(\Lambda_{k}(x+y)\right)}{\rho}\right) t_{k}^{\frac{1}{p_{k}}}\right\} & =\sup _{k \geq 1}\left\{M_{k}\left(\frac{q\left(\Lambda_{k}(x+y)\right)}{\rho_{1}+\rho_{2}}\right) t_{k}^{\frac{1}{p_{k}}}\right\} \\
& \leq\left(\frac{\rho_{1}}{\rho_{1}+\rho_{2}}\right) \sup _{k \geq 1}\left[M_{k}\left(\frac{q\left(\Lambda_{k}(x)\right)}{\rho_{1}}\right) t_{k}^{\frac{1}{p_{k}}}\right] \\
& +\left(\frac{\rho_{2}}{\rho_{1}+\rho_{2}}\right) \sup _{k \geq 1}\left[M_{k}\left(\frac{q\left(\Lambda_{k}(y)\right)}{\rho_{2}}\right) t_{k}^{\frac{1}{p_{k}}}\right] \\
& \leq 1
\end{aligned}
$$

and thus

$$
\begin{aligned}
g(x+y) & =q\left(x_{1}+y_{1}\right) \\
& +\inf \left\{\left(\rho_{1}+\rho_{2}\right)^{\frac{p_{k}}{H}}: \sup _{k \geq 1}\left\{M_{k}\left(\frac{q\left(\Lambda_{k}(x)+\Lambda_{k}(y)\right)}{\rho}\right)\right\} t_{k}^{\frac{1}{p_{k}}} \leq 1, \rho>0\right\} \\
& \leq q\left(x_{1}\right)+\inf \left\{\left(\rho_{1}\right)^{\frac{p_{k}}{H}}: \sup _{k \geq 1}\left\{M_{k}\left(\frac{q\left(\Lambda_{k}(x)\right)}{\rho_{1}}\right)\right\} t_{k}^{\frac{1}{p_{k}}} \leq 1, \rho>0\right\} \\
& +q\left(y_{1}\right)+\inf \left\{\left(\rho_{2}\right)^{\frac{p_{k}}{H}}: \sup _{k \geq 1}\left\{M_{k}\left(\frac{q\left(\Lambda_{k}(y)\right)}{\rho_{2}}\right)\right\} t_{k}^{\frac{1}{p_{k}}} \leq 1, \rho>0\right\}
\end{aligned}
$$




$$
\leq \mathrm{g}(\mathrm{x})+\mathrm{g}(\mathrm{y})
$$

(iv) Finally, we prove that the scalar multiplication is continuous. Let $\mu$ be any complex number. By definition,

$$
\begin{aligned}
g(\mu x) & =q\left(\mu x_{1}\right)+\inf \left\{\rho^{\frac{p_{k}}{H}}: \sup _{k \geq 1}\left\{M_{k}\left(\frac{q\left(\mu \Lambda_{k}(x)\right)}{\rho}\right)\right\} t_{k}^{\frac{1}{p_{k}}} \leq 1, \quad \rho>0\right\} \\
& =|\mu| q\left(x_{1}\right)+\inf \left\{(|\lambda| r)^{\frac{p_{k}}{H}}: \sup _{k \geq 1}\left\{M_{k}\left(\frac{q\left(\Lambda_{k}(x)\right)}{r}\right)\right\} t_{k}^{\frac{1}{p_{k}}} \leq 1, \quad r>0\right\},
\end{aligned}
$$

where $r=\frac{\rho}{|\mu|}$. Hence $l_{\infty}\{\mathcal{M}, \Lambda, p, q\}$ is a paranormed space.

Theorem 3 For any Musielak-Orlicz function $\mathcal{M}=\left(M_{k}\right)$ and $p=\left(p_{k}\right) \in l_{\infty}$, then the spaces $\mathfrak{c}_{0}\{\mathcal{M}, \Lambda, p, q\}, c\{\mathcal{M}, \Lambda, p, q\}$ and $l_{\infty}\{\mathcal{M}, \Lambda, p, q\}$ are complete paranormed spaces paranormed by $\mathrm{g}$.

Proof. Suppose $\left(x^{\mathfrak{n}}\right)$ is a Cauchy sequence in $l_{\infty}\{\mathcal{M}, \wedge, p, q\}$, where $x^{\mathfrak{n}}=$ $\left(x_{k}^{n}\right)_{k=1}^{\infty}$ for all $n \in \mathbb{N}$. So that $g\left(x^{i}-x^{j}\right) \rightarrow 0$ as $i, j \rightarrow \infty$. Suppose $\epsilon>0$ is given and let $s$ and $x_{0}$ be such that $\frac{\epsilon}{s x_{0}}>0$ and $M_{k}\left(\frac{s x_{0}}{2}\right) \geq \sup _{k \geq 1}\left(p_{k}\right)^{t_{k}}$. Since $g\left(x^{i}-x^{j}\right) \rightarrow 0$, as $i, j \rightarrow \infty$ which means that there exists $n_{0} \in \mathbb{N}$ such that

$$
g\left(x^{i}-x^{j}\right)<\frac{\epsilon}{s x_{0}}, \text { for all } i, j \geq n_{0} .
$$

This gives $g\left(x_{1}^{i}-x_{1}^{j}\right)<\frac{\epsilon}{s x_{0}}$ and

$$
\inf \left\{\rho^{\frac{p_{k}}{H}}: \sup _{k \geq 1}\left\{M_{k}\left(\frac{q\left(\Lambda_{k}\left(x^{i}-x^{j}\right)\right)}{\rho}\right) t_{k}^{\frac{1}{p_{k}}}\right\} \leq 1, \rho>0\right\}<\frac{\epsilon}{s x_{0}} .
$$

It shows that $\left(x_{1}^{i}\right)$ is a Cauchy sequence in $X$. Therefore $\left(x_{1}^{i}\right)$ is convergent in $X$ because $X$ is complete. Suppose $\lim _{i \rightarrow \infty} x_{1}^{i}=x_{1}$ then $\lim _{j \rightarrow \infty} g\left(x_{1}^{i}-x_{1}^{j}\right)<\frac{\epsilon}{s x_{0}}$, we get

$$
g\left(x_{1}^{i}-x_{1}\right)<\frac{\epsilon}{s x_{0}} .
$$

Thus, we have

$$
M_{k}\left(\frac{q\left(\Lambda_{k}\left(x^{i}-x^{j}\right)\right)}{g\left(x^{i}-x^{j}\right)}\right) t_{k}^{\frac{1}{p_{k}}} \leq 1
$$

This implies that

$$
M_{k}\left(\frac{q\left(\Lambda_{k}\left(x^{i}-x^{j}\right)\right)}{g\left(x^{i}-x^{j}\right)}\right) \leq\left(p_{k}\right)^{t_{k}} \leq M_{k}\left(\frac{s x_{0}}{2}\right)
$$


and thus

$$
q\left(\Lambda_{k}\left(x^{i}-\chi^{j}\right)\right)<\frac{s x_{0}}{2} \cdot \frac{\epsilon}{s x_{0}}<\frac{\epsilon}{2}
$$

which shows that $\left(\Lambda_{k}\left(x^{i}\right)\right)$ is a Cauchy sequence in $X$ for all $k \in \mathbb{N}$. Therefore, $\left(\Lambda_{k}\left(x^{i}\right)\right)$ converges in $X$. Suppose $\lim _{i \rightarrow \infty} \Lambda_{k}\left(x^{i}\right)=y$ for all $k \in \mathbb{N}$. Also, we have $\lim _{i \rightarrow \infty} \Lambda_{k}\left(x_{2}^{i}\right)=y_{1}-x_{1}$. On repeating the same procedure, we obtain $\lim _{i \rightarrow \infty} \Lambda_{k}\left(x_{k+1}^{i}\right)=y_{k}-x_{k}$ for all $k \in \mathbb{N}$. Therefore by continuity of $\left(M_{k}\right)$, we get

$$
\lim _{j \rightarrow \infty} \sup _{k \geq 1} M_{k}\left(\frac{q\left(\Lambda_{k}\left(x^{i}-x^{j}\right)\right)}{\rho}\right) t_{k}^{\frac{1}{p_{k}}} \leq 1
$$

so that

$$
\sup _{k \geq 1} M_{k}\left(\frac{q\left(\Lambda_{k}\left(x^{i}-x^{j}\right)\right)}{\rho}\right) t_{k}^{\frac{1}{p_{k}}} \leq 1
$$

Let $i \geq n_{0}$ and taking infimum of each $\rho$ 's, we have

$$
g\left(x^{i}-x\right)<\epsilon .
$$

So $\left(x^{i}-x\right) \in l_{\infty}\{\mathcal{M}, \Lambda, p, q\}$. Hence $x=x^{i}-\left(x^{i}-x\right) \in l_{\infty}\{\mathcal{M}, \Lambda, p, q\}$, since $l_{\infty}\{\mathcal{M}, \Lambda, p, q\}$ is a linear space. Hence, $l_{\infty}\{\mathcal{M}, \Lambda, p, q\}$ is a complete paranormed space. Similarly, we can prove the spaces $c_{0}\{\mathcal{M}, \Lambda, p, q\}$ and $c\{\mathcal{M}, \Lambda, p, q\}$ are complete paranormed spaces.

Theorem 4 If $0<p_{k} \leq r_{k}<\infty$ for each $k$, then

$$
\mathrm{Z}\{\mathcal{M}, \wedge, p, q\} \subseteq \mathrm{Z}\{\mathcal{M}, \wedge, \mathrm{r}, \mathrm{q}\}
$$

for $\mathrm{Z}=\mathrm{c}_{0}$ and $\mathrm{c}$.

Proof. Let $x=\left(x_{k}\right) \in c\{\mathcal{M}, \Lambda, p, q\}$. Then there exists some $\rho>0$ and $L \in X$ such that

$$
M_{k}\left(\frac{q\left(\Lambda_{k}(x)-L\right)}{\rho}\right)^{p_{k}} t_{k} \rightarrow 0 \text { as } k \rightarrow \infty .
$$

This implies that

$$
M_{k}\left(\frac{q\left(\Lambda_{k}(x)-L\right)}{\rho}\right)<\epsilon, \quad(0<\epsilon<1)
$$


for sufficiently large $k$. Hence we get

$$
M_{k}\left(\frac{q\left(\Lambda_{k}(x)-L\right)}{\rho}\right)^{r_{k}} t_{k} \leq M_{k}\left(\frac{q\left(\Lambda_{k}(x)-L\right)}{\rho}\right)^{p_{k}} t_{k} \rightarrow 0 \text { as } k \rightarrow \infty .
$$

This implies that $x=\left(x_{k}\right) \in c\{\mathcal{M}, \wedge, r, q\}$. This completes the proof. Similarly, we can prove for the case $Z=c_{0}$.

Theorem 5 Suppose $\mathcal{M}^{\prime}=\left(M_{k}^{\prime}\right)$ and $\mathcal{M}^{\prime \prime}=\left(M_{k}^{\prime \prime}\right)$ are Musielak-Orlicz functions satisfying the $\Delta_{2}$-condition then we have the following results:

(i) if $\left(\mathrm{p}_{\mathrm{k}}\right) \in \mathrm{l}_{\infty}$ then $\mathrm{Z}\left\{\mathcal{M}^{\prime}, \Lambda, p, q\right\} \subseteq \mathrm{Z}\left\{\mathcal{M}^{\prime \prime} \circ \mathcal{M}^{\prime}, \Lambda, p, q\right\}$ for $Z=c, c_{0}$ and $l_{\infty}$.

(ii) $Z\left\{\mathcal{M}^{\prime}, \Lambda, p, q\right\} \cap Z\left\{\mathcal{M}^{\prime \prime}, \Lambda, p, q\right\} \subseteq Z\left\{\mathcal{M}^{\prime}+\mathcal{M}^{\prime \prime}, \Lambda, p, q\right\}$ for $Z=c, c_{0}$ and $l_{\infty}$.

Proof. If $x=\left(x_{k}\right) \in c_{0}\{\mathcal{M}, \Lambda, p, q\}$ then there exists some $\rho>0$ such that

$$
\left\{M_{k}^{\prime}\left(\frac{q\left(\Lambda_{k}(x)\right)}{\rho}\right)\right\}^{p_{k}} t_{k} \rightarrow 0 \text { as } k \rightarrow \infty
$$

Suppose

$$
y_{k}=M_{k}^{\prime}\left(\frac{q\left(\Lambda_{k}(x)\right)}{\rho}\right) \text { for all } k \in \mathbb{N} \text {. }
$$

Choose $\delta>0$ be such that $0<\delta<1$, then for $y_{k} \geq \delta$ we have $y_{k}<\frac{y_{k}}{\delta}<1+\frac{y_{k}}{\delta}$. Now $\left(M_{k}^{\prime \prime}\right)$ satisfies $\Delta_{2}$-condition so that there exists $J \geq 1$ such that

$$
M_{k}^{\prime \prime}\left(y_{k}\right)<\frac{J y_{k}}{2 \delta} M_{k}^{\prime \prime}(2)+\frac{J y_{k}}{2 \delta} M_{k}^{\prime \prime}(2)=\frac{J y_{k}}{\delta} M_{k}^{\prime \prime}(2) .
$$

We obtain

$$
\begin{aligned}
& {\left[\left(M_{k}^{\prime \prime} \circ M_{k}^{\prime}\right)\left(\frac{q\left(\Lambda_{k}(x)\right)}{\rho}\right)\right]^{p_{k}} t_{k}=\left[M_{k}^{\prime \prime}\left\{M_{k}^{\prime}\left(\frac{q\left(\Lambda_{k}(x)\right)}{\rho}\right)\right\}\right]^{p_{k}} t_{k}=\left[M_{k}^{\prime \prime}\left(y_{k}\right)\right]^{p_{k}} t_{k}} \\
& \leq \max \left\{\sup _{k}\left(\left[M_{k}^{\prime \prime}(1)\right]^{p_{k}}\right), \sup _{k}\left(\left[k M_{k}^{\prime \prime}(2) \delta^{-1}\right]^{p_{k}}\right)\right\}\left[y_{k}\right]^{p_{k}} t_{k} \rightarrow 0, \text { as } k \rightarrow \infty .
\end{aligned}
$$

Similarly, we can prove the other cases. 
(ii) Suppose $x=\left(x_{k}\right) \in c_{0}\left\{M_{k}^{\prime}, \wedge, p, q\right\} \cap c_{0}\left\{M_{k}^{\prime \prime}, \wedge, p, q\right\}$, then there exist $\rho_{1}, \rho_{2}>0$ such that

$$
\left\{\left(M_{k}^{\prime}\left(\frac{\mathrm{q}\left(\Lambda_{\mathrm{k}}(\mathrm{x})\right)}{\rho_{1}}\right)\right)^{\mathrm{p}_{\mathrm{k}}} \mathrm{t}_{\mathrm{k}}\right\} \rightarrow 0, \text { as } \mathrm{k} \rightarrow \infty
$$

and

$$
\left\{\left(M_{k}^{\prime \prime}\left(\frac{\mathrm{q}\left(\Lambda_{\mathrm{k}}(\mathrm{x})\right)}{\rho_{2}}\right)\right)^{\mathrm{p}_{\mathrm{k}}} \mathrm{t}_{\mathrm{k}}\right\} \rightarrow 0, \text { as } \mathrm{k} \rightarrow \infty .
$$

Let $\rho=\max \left\{\rho_{1}, \rho_{2}\right\}$. The remaining proof follows from the inequality

$$
\begin{aligned}
\left\{\left[\left(M_{k}^{\prime}+M_{k}^{\prime \prime}\right)\left(\frac{q\left(\Lambda_{k}(x)\right)}{\rho}\right)\right]^{p_{k}} t_{k}\right\} \leq & D\left\{\left[M_{k}^{\prime}\left(\frac{q\left(\Lambda_{k}(x)\right)}{\rho_{1}}\right)\right]^{p_{k}} t_{k}\right. \\
& \left.+\left[M_{k}^{\prime \prime}\left(\frac{q\left(\Lambda_{k}(x)\right)}{\rho_{2}}\right)\right]^{p_{k}} t_{k}\right\} .
\end{aligned}
$$

Hence $c_{0}\left\{M_{k}^{\prime}, \Lambda, p, q\right\} \cap c_{0}\left\{M_{k}^{\prime \prime}, \Lambda, p, q\right\} \subseteq c_{0}\left\{M_{k}^{\prime}+M_{k}^{\prime \prime}, \Lambda, p, q\right\}$. Similarly we can prove the other cases.

Theorem 6 (i) If $0<\inf p_{k} \leq p_{k}<1$, then $l_{\infty}\{\mathcal{M}, \Lambda, p, q\} \subset l_{\infty}\{\mathcal{M}, \Lambda, q\}$.

(ii) If $1 \leq p_{k} \leq \sup p_{k}<\infty$, then $l_{\infty}\{\mathcal{M}, \wedge, q\} \subset l_{\infty}\{\mathcal{M}, \wedge, p, q\}$.

Proof. (i) Let $x=\left(x_{k}\right) \in l_{\infty}\{\mathcal{M}, \Lambda, p, q\}$. Since $0<\inf p_{k} \leq 1$, we have

$$
\sup _{k}\left\{\left[M_{k}\left(\frac{q\left(\Lambda_{k}(x)\right)}{\rho_{2}}\right)\right]\right\} \leq \sup _{k}\left\{\left[M_{k}\left(\frac{q\left(\Lambda_{k}(x)\right)}{\rho_{2}}\right)\right]^{p_{k}} t_{k}\right\}
$$

and hence $x=\left(x_{k}\right) \in l_{\infty}\{\mathcal{M}, \Lambda, q\}$.

(ii) Let $p_{k} \geq 1$ for each $k$ and $\sup _{k} p_{k}<\infty$. Let $x=\left(x_{k}\right) \in l_{\infty}\{\mathcal{M}, \wedge, q\}$, then for each $\epsilon, \quad 0<\epsilon<1$, there exists a positive integer $n_{0} \in \mathbb{N}$ such that

$$
\sup _{k}\left\{M_{k}\left(\frac{q\left(\Lambda_{k}(x)\right)}{\rho}\right)\right\} \leq \epsilon<1 .
$$

This implies that

$$
\sup _{k}\left\{\left[M_{k}\left(\frac{q\left(\Lambda_{k}(x)\right)}{\rho}\right)\right]^{p_{k}} t_{k}\right\} \leq \sup _{k}\left\{M_{k}\left(\frac{q\left(\Lambda_{k}(x)\right)}{\rho}\right)\right\} .
$$

Thus $x=\left(x_{k}\right) \in l_{\infty}\{\mathcal{M}, \Lambda, p, q\}$ and this completes the proof. 


\section{References}

[1] T. Bilgin, Some new difference sequences spaces defined by an Orlicz function, Filomat, 17 (2003), 1-8.

[2] A. Esi, Some new paranormed sequence spaces defined by Orlicz function, International Journal of Science, Environment and Technology, 1 (2012), $49-55$.

[3] J. Lindenstrauss and L. Tzafriri, On Orlicz sequence spaces, Israel J. Math., 10 (1971), 379-390.

[4] L. Maligranda, Orlicz spaces and interpolation, Seminars in Mathematics 5, Polish Academy of Science, 1989.

[5] M. Mursaleen, Generalized spaces of difference sequences, J. Math. Anal. Appl., 203 (1996), 738-745.

[6] M. Mursaleen and A. K. Noman, On some new sequence spaces of non absolute type related to the spaces $l_{p}$ and $l_{\infty}$ I, Filomat, 25 (2011), 33-51.

[7] M. Mursaleen and A. K. Noman, On some new sequence spaces of non absolute type related to the spaces $l_{p}$ and $l_{\infty}$ II, Math. Commun., 16 (2011), 383-398.

[8] J. Musielak, Orlicz spaces and modular spaces, Lecture Notes in Mathematics, 1034 (1983).

[9] K. Raj, A. K. Sharma and S. K. Sharma, A Sequence space defined by Musielak-Orlicz functions, Int. J. Pure Appl. Math., 67 (2011), 475-484.

[10] K. Raj, S. K. Sharma and A. K. Sharma, Some difference sequence spaces in n-normed spaces defined by Musielak-Orlicz function, Armenian $J$. Math., 3 (2010), 127-141.

[11] K. Raj and S. K. Sharma, Some sequence spaces in 2-normed spaces defined by Musielak-Orlicz function, Acta. Univ. Saprientia Math., 3 (2011), 97-109.

[12] K. Raj and S. K. Sharma, Some multiplier sequence spaces defined by a Musielak-Orlicz function in n-normed spaces, New Zealand J. Math., 42 (2012), 45-56. 
[13] B. C. Tripathy and B. Sarma, Some Some classes of difference paranormed sequence spaces defined by Orlicz function, Thai J. Math., 3 (2005), 209218.

[14] A. Wilansky, Summability through Functional Analysis, North- Holland Math. Stud. 85 (1984). 